\title{
Series Solutions of Boundary Layer Flow of a Micropolar Fluid Near the Stagnation Point Towards a Shrinking Sheet
}

\author{
Sohail Nadeem ${ }^{\mathrm{a}}$, Saeid Abbasbandy ${ }^{\mathrm{b}}$, and Majid Hussain ${ }^{\mathrm{a}}$ \\ a Department of Mathematics, Quaid-i-Azam University 45320, Islamabad 44000, Pakistan \\ b Department of Mathematics, Imam Khomeini International University, Ghazvin 34149-16818, Iran \\ Reprint requests to S. N.; e-mail: snqau@ hotmail.com
}

Z. Naturforsch. 64a, 575 - 582 (2009); received September 16, 2008 / revised November 6, 2008

\begin{abstract}
An analysis has been carried out to obtain the series solution of boundary layer flow of a micropolar fluid towards a shrinking sheet. The governing equations of micropolar fluid are simplified using suitable similarity transformations and then solved by homotopy analysis method (HAM). The convergence of the HAM solutions has been obtained by using homotopy-pade approximation. The effects of various parameters such as porosity parameter $R$, the ratio $\lambda$ and the microinertia $K$ on the velocity and microinertia profiles as well as local skin friction coefficient are presented graphically and in tabulated form.
\end{abstract}

Key words: Micropolar Fluid; Stagnation Point; Shrinking Sheet; Boundary Layer Flow

\section{Introduction}

A phenomena in which all solid bodies moving in a fluid and describing the fluid motion near the stagnation region is known as stagnation flow.

The boundary layer flows which describe the stagnation flows and stretching surface arises in a number of practical applications. Such applications include rotating blades, cooling of silicon wafers, and extrusion of a polymer in a melt spinning process. The extrudate from a die is generally drawn and simultaneously stretched into a sheet which is then solidified through quenching or gradual cooling by direct contact with water.

Stagnation point flow towards a stretching surface has been discussed by many researchers. Mahapatra and Gupta [1] have studied the magnetohydrodynamic (MHD) stagnation point flow towards a stretching sheet. They found that the velocity at a point decreases/increases with the increase in the magnetic field when the free stream velocity is less/greater than the stretching velocity. Nazar et al. [2] discussed the unsteady boundary layer flow in the region of stagnation point on a stretching surface. Later on Lok et al. [3] extended the idea of Nazar et al. [2] for mixed conviction flow. Mixed convection flow near a nonorthogonal stagnation point towards a stretching vertical plate has been discussed by Lok et al. [4]. They considered both the assisting and opposing flows and found that the flow has an inverted boundary layer suc- tion when the stretching velocity of the surface exceeds the stagnation velocity of the free stream. Recently, Wang [5] has discussed the off-centered stagnation flow towards a rotating disc. He found that the non-alignment complicates the flow field and surface shear, but does not effect the torque. In spite of all these very little has been discussed about the shrinking sheet when the velocity on the boundary is towards a fixed point. The shrinking film and viscous flow due to shrinking sheet have been discussed by Miklavic and Wang [6] and Wang [7]. Hayat et al. [8] extended the idea of Wang [5] and discussed the rotating flow over a shrinking surface. Recently, Nadeem and Awais [9] examined the thin film flow of an unsteady shrinking sheet through porous medium with variable viscosity. More recently, Wang [10] has discussed the stagnation flow towards a shrinking sheet. $\mathrm{He}$ observed that the non-alignment of the stagnation flow and shrinking sheet complicates the flow structure. To the best of authors knowledge the stagnation point flow towards a shrinking sheet has not been discussed for micropolar fluid. Micropolar fluid was proposed by Eringen [11], because the classical NavierStokes theory does not describe adequately the flow properties of either polymeric fluids or certain naturally occurring fluids such as animal blood. Micropolar fluids have received considerable attention due to their applications in a number of processes that occur in industry. Such applications include the extrusion of 
polymer fluids solidification of liquid crystals, cooling of a metallic plate in a bath, animal bloods, exotic lubricants, and colloidal suspension. In the present paper we have discussed the stagnation point flow of a micropolar fluid towards a shrinking surface. The governing equations are highly nonlinear which have been simplified by similarity transformations and then find the analytic solutions by means of Homotopy analysis method (HAM). HAM has been successfully applied to many nonlinear problems [12-32]. Graphical results are presented for different parameters appearing in the present model.

\section{Mathematical Formulation}

Let us consider an incompressible micropolar fluid near a stagnation point toward a porous shrinking plate. We are considering a Cartesian coordinate system such that shrinking sheet is taken along the $x$-axis while the fluid is along the $y$-axis. The governing boundary layer equations for the micropolar fluid are

$$
\begin{gathered}
\frac{\partial u}{\partial x}+\frac{\partial v}{\partial y}=0 \\
u \frac{\partial u}{\partial x}+v \frac{\partial u}{\partial y}=u_{e} \frac{d u_{e}}{d x}+\left(v+\frac{k}{\rho}\right) \frac{\partial^{2} u}{\partial y^{2}}+\frac{k}{\rho} \frac{\partial N}{\partial y} \\
u \frac{\partial N}{\partial x}+v \frac{\partial N}{\partial y}=\frac{\gamma}{\rho j} \frac{\partial^{2} N}{\partial y^{2}}-\frac{k}{\rho j}\left(2 N+\frac{\partial u}{\partial y}\right)
\end{gathered}
$$

The corresponding boundary conditions for the problem are

$$
\begin{aligned}
& u_{e}(x, 0)=-b x, \quad v(x, 0)=-v_{0}, \\
& N(x, 0)=-n \frac{\partial u}{\partial y}, \\
& u(x, y) \longrightarrow u_{e}(x)=a x, \\
& N(x, y) \longrightarrow 0, \text { as } y \longrightarrow \infty .
\end{aligned}
$$

In the above equations, $u$ and $v$ are the velocity components along $x$ - and $y$-axis, $u_{e}(=a x)$ is the free stream velocity, $N$ is the microrotation velocity, $v$ is the viscosity, $\rho$ is the density, $n \in[0,1]$ is a constant, $a, b$ both are positive constants and $j, \gamma$ and $k$ are the microinertia per unit mass, spin gradient viscosity and vortex viscosity, respectively, which are assumed to be constant. Here $\gamma$ is given by [33]

$$
\gamma=\left(\frac{\mu+k}{2}\right) j,
$$

and here we consider $j=v / b$ as a reference length.
We introduce the non-dimensional quantities

$$
\begin{aligned}
& u=b x f^{\prime}(\eta), \quad v=-(b v)^{\frac{1}{2}} f(\eta), \\
& N=\left(\frac{b^{3}}{v}\right)^{\frac{1}{2}} x g(\eta), \quad \eta=\left(\frac{b}{v}\right)^{\frac{1}{2}} y, \\
& \Psi=(b v)^{\frac{1}{2}} x f(\eta) .
\end{aligned}
$$

Making use of (6), the conditions of incompressibility is satisfied and (2), (3) along with boundary conditions (4), (5) take the form

$$
\begin{aligned}
& (1+K) f^{\prime \prime \prime}+f f^{\prime \prime}-f^{2}+K g^{\prime}+\lambda^{2}=0 \\
& \left(1+\frac{K}{2}\right) g^{\prime \prime}+f g^{\prime}-f^{\prime} g-K\left(2 g+f^{\prime \prime}\right)=0 \\
& f(0)=\frac{v_{0}}{(b v)^{\frac{1}{2}}}=R, \quad f^{\prime}(0)=-1 \\
& g(0)=-n f^{\prime \prime}(0), \quad f^{\prime}(\eta)=\lambda \\
& g(\eta)=0 \text { as } \eta \longrightarrow \infty
\end{aligned}
$$

where $\lambda=\frac{a}{b}(>0), K=\frac{k}{\mu}$, and $R=v_{0} /(b v)^{\frac{1}{2}}$.

For micropolar boundary layer flow, the wall skin friction $\tau_{w}$ is given by

$$
\tau_{w}=\left[(\mu+k) \frac{\partial u}{\partial y}+k N\right]_{y=0} .
$$

The non-dimensional form of above equation can be written as

$$
C_{f} R_{e_{x}}^{\frac{1}{2}}=(1+K) f^{\prime \prime}(0)+K g(0)
$$

where $u_{W}(x)=b x$ is the characteristic velocity.

Equations (8) and (9) are coupled nonlinear differential equations. To find the analytic solutions we use the Homotopy analysis method (HAM).

\section{Solution of the Problem}

According to HAM procedure, one can choose any type of initial guess. Thus we take

$$
\begin{aligned}
& f_{0}(\eta)=R+(\lambda \eta)+(1+\lambda)\left(\mathrm{e}^{-\eta}-1\right), \\
& g_{0}(\eta)=-n \cdot f_{0}^{\prime \prime}(0) \mathrm{e}^{-\eta}
\end{aligned}
$$

as the initial guesses and

$$
L_{f}[f(\eta)]=\frac{\partial^{3} f}{\partial \eta^{3}}-\frac{\partial f}{\partial \eta},
$$




$$
L_{g}[g(\eta)]=\frac{\partial^{2} g}{\partial \eta^{2}}-g
$$

as the auxiliary linear operators, which have the following property:

$$
\begin{aligned}
& L_{f}\left[c_{1} \mathrm{e}^{-\eta}+c_{2} \mathrm{e}^{\eta}+c_{3}\right]=0, \\
& L_{g}\left[c_{4} \mathrm{e}^{-\eta}+c_{5} \mathrm{e}^{\eta}\right]=0 .
\end{aligned}
$$

In the above equations $c_{1}, c_{2}, c_{3}, c_{4}$, and $c_{5}$ are constants. From (8) and (9), we can define the zeroth-order deformation equations as

$$
\begin{gathered}
(1-p) L_{f}\left[\hat{f}(\eta ; p)-f_{0}(\eta)\right]=p \hbar_{f} N_{f}[\hat{f}(\eta ; p)], \\
(1-p) L_{g}\left[\hat{g}(\eta ; p)-g_{0}(\eta)\right]=p \hbar_{g} N_{g}[\hat{g}(\eta ; p)], \\
\hat{f}(0 ; p)=R, \quad \frac{\partial \hat{f}}{\partial \eta}(0 ; p)=-1, \\
\hat{g}(0 ; p)=-n \frac{\partial^{2} \hat{f}}{\partial \eta^{2}}(0), \\
\frac{\partial \hat{f}}{\partial \eta}(\eta)=\lambda, \quad \hat{g}(\eta) \longrightarrow 0 \text { as } \eta \longrightarrow \infty,
\end{gathered}
$$

where $\hbar_{f}$ and $\hbar_{g}$ are non-zero auxiliary parameters, $p \in$ $[0,1]$ is the embedding parameter and

$$
\begin{aligned}
N_{f}[\tilde{f}(\eta, p)]= & (1+K) \frac{\partial^{3} \hat{f}}{\partial \eta^{3}}+\hat{f} \frac{\partial^{2} \hat{f}}{\partial \eta^{2}}+K \frac{\partial \hat{g}}{\partial \eta} \\
& -\left(\frac{\partial \hat{f}}{\partial \eta}\right)^{2}+\lambda^{2} \\
N_{g}[\hat{g}[(\eta ; p)]= & \left(1+\frac{K}{2}\right) \frac{\partial^{2} \hat{g}}{\partial \eta^{2}}+\hat{f} \frac{\partial \hat{g}}{\partial \eta} \\
& -\hat{g} \frac{\partial \hat{f}}{\partial \eta}-K\left(2 \hat{g}+\frac{\partial^{2} \hat{f}}{\partial \eta^{2}}\right) .
\end{aligned}
$$

Obviously

$$
\begin{aligned}
& \hat{f}(\eta, 0)=f_{0}(\eta) \text { and } \hat{g}(\eta, 0)=g_{0}(\eta), \\
& \hat{f}(\eta, 1)=f(\eta) \text { and } \hat{g}(\eta, 1)=g(\eta) .
\end{aligned}
$$

As $p$ goes from 0 to $1, \hat{f}(\eta, p)$ and $\hat{g}(\eta, p)$ vary from initial guesses $f_{0}(\eta)$ and $g_{0}(\eta)$ to final solutions $f(\eta)$, and $g(\eta)$, respectively. Making the assumption that the auxiliary parameters $\hbar_{f}$ and $\hbar_{g}$ are so properly chosen that the Taylor series of $f(\eta ; p)$ and $g(\eta ; p)$ expanded with respect to embedding parameters converges at $p=1$. Thus by making use of (25) and (26), we write

$$
f(\eta)=f_{0}(\eta)+\sum_{m=1}^{\infty} f_{m}(\eta)
$$

$$
g(\eta)=g_{0}(\eta)+\sum_{m=1}^{\infty} g_{m}(\eta)
$$

where

$$
\begin{aligned}
& f_{m}(\eta)=\left.\frac{1}{m !} \frac{\partial^{m} \hat{f}(\eta, p)}{\partial p^{m}}\right|_{p=0}, \\
& g_{m}(\eta)=\left.\frac{1}{m !} \frac{\partial^{m} \hat{g}(\eta ; p)}{\partial p^{m}}\right|_{p=0} .
\end{aligned}
$$

Differentiating the zeroth-order deformation equations (19) and (20) and boundary conditions (21) and (22) $m$ times with respect to $p$, then dividing by $m$ ! and finally setting $p=0$, we get the mth-order deformation equations as

$$
\begin{gathered}
L_{f}\left[f_{m}(\eta)-\chi_{m} f_{m-1}(\eta)\right]=\hbar_{f} R_{f, m}(\eta), \\
L_{g}\left[g_{m}(\eta)-\chi_{m} g_{m-1}(\eta)\right]=\hbar_{g} R_{g, m}(\eta), \\
f_{m}(0)=0, \quad f_{m}^{\prime}(0)=0, \quad g_{m}(0)=-n f_{m}^{\prime \prime}(0), \\
f_{m}^{\prime}(\eta)=0, \quad g(\eta)=0, \text { as } \eta \longrightarrow \infty
\end{gathered}
$$

where

$$
\begin{gathered}
R_{f, m}(\eta)=(1+K) \frac{\partial^{3} f_{m-1}}{\partial \eta^{3}}+\sum_{i=0}^{m-1} f_{i} \frac{\partial^{2} f_{m-i-1}}{\partial \eta^{2}} \\
+K \frac{\partial g_{m-1}}{\partial \eta}-\sum_{i=0}^{m-1} \frac{\partial f_{i}}{\partial \eta} \frac{\partial f_{m-i-1}}{\partial \eta}+\lambda^{2}\left(1-\chi_{m}\right), \\
R_{g, m}(\eta)=\left(1+\frac{K}{2}\right) \frac{\partial^{2} g_{m-1}}{\partial \eta^{2}}+\sum_{i=0}^{m-1} f_{i} \frac{\partial g_{m-1-i}}{\partial \eta} \\
-\sum_{i=0}^{m-1} g_{i} \frac{\partial f_{m-1-i}}{\partial \eta}-K\left(2 g_{m-1}+\frac{\partial^{2} f_{m-1}}{\partial \eta^{2}}\right), \\
\chi_{m}= \begin{cases}0, & m=1, \\
1, & m>1 .\end{cases}
\end{gathered}
$$

With the help of MATHEMATICA, the solution of (31) and (32) subject to boundary conditions (33) and (34) we can write as

$$
\begin{aligned}
& f(\eta)=A_{0,0}+A_{1,0} \eta+\sum_{k=0}^{\infty} \sum_{m=1}^{\infty} A_{k, m} \eta^{k} \mathrm{e}^{-m \eta} \\
& g(\eta)=\sum_{k=0}^{\infty} \sum_{m=0}^{\infty} B_{k, m} \eta^{k} \mathrm{e}^{-m \eta}
\end{aligned}
$$

where $A_{0,0}, A_{1,0}, A_{k, m}$ and $B_{k, m}$ are constants. 

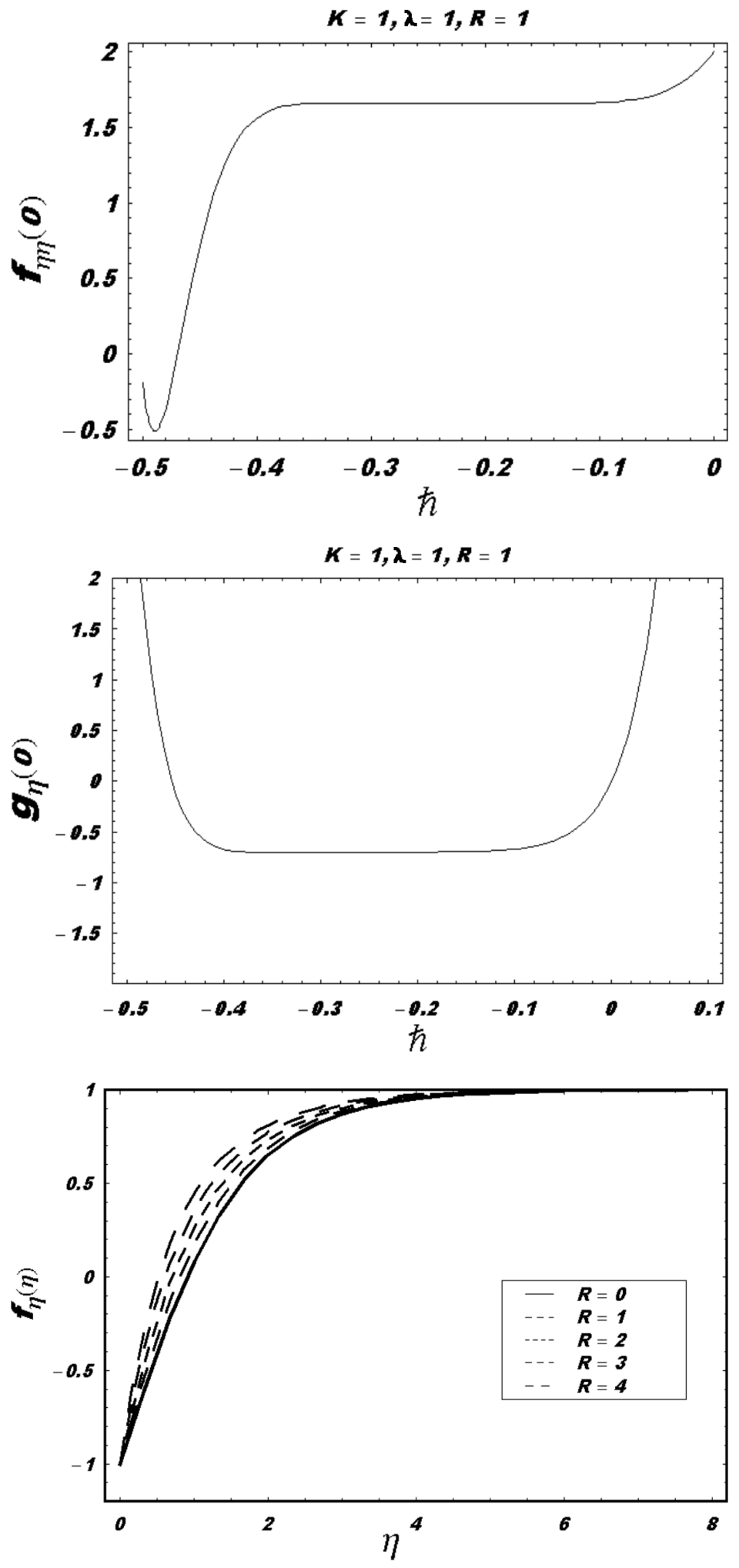

Fig. $1 . \hbar$ curve for $f^{\prime \prime}(0) 25$ th-order of approximation.

Fig. 2. $\hbar$ curve for $g^{\prime}(0) 25$ th-order of approximation.

Fig. 3. Velocity field for different values of $R$ when $K=1$ and $n=0$. 


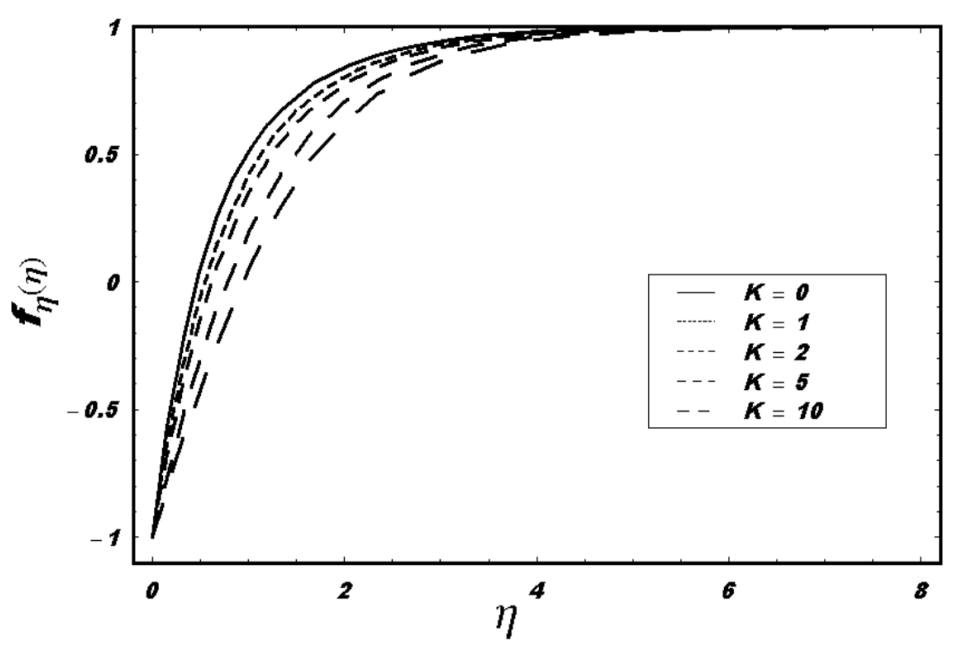

Fig. 4. Velocity field for different values of $K$ when $R=1$ and $n=0$.

\section{Discussion}

Equations (8) and (9) with boundary condition (10) are solved analytically by HAM. In order to assess the convergence of the HAM solutions, the homotopypade approximation is applied and the results are tabulated in Table 1. Also the $\hbar$ curves are plotted for both $f$ and $g$ to show the convergence region which are shown in Figures 1 and 2. Thus we conclude from Table 1, Figures 1 and 2 that homotopy-pade approximation and $\hbar$-curves of HAM give the similar behavior (convergent).

The analytical results for the velocity, microrotation and skin friction coefficient have been obtained for $n=0$ (strong concentration) and $n=1 / 2$ (weak concentration) for different values of $\lambda, K$ and $R$.

In Table 2, the coefficient of skin friction is computed. It is found that for a fix value of $\lambda$ and with the increase in $R$ and $K$, the coefficient of skin friction increases and for any fixed $R$ and $K$ with the increase in $\lambda$, the coefficient of skin friction decreases for $\lambda \leq 2$ while for $\lambda>2$ it is increasing.

The non-dimensional velocity versus $\eta$ for different values of $R$ and $K$ are plotted in Figure 3 and 4. It is observed that with the increase in $\mathrm{R}$ the magnitude of velocity increases while the magnitude of velocity decreases with the increase in $K$. Further we say that suction causes the reduction of the boundary layer. It is also shown that for large values of $R$, the solution is not stable (see Fig. 5). In other words for larger shrinking parameter our solutions are unstable. It means that non-uniqueness and non-existence of the solution happens due to unique character of the
Table. 1. Convergence of series solutions using homotopypade approximation.

\begin{tabular}{ccc}
\hline Homotopy-Pade approximation & $-f^{\prime \prime}(0)$ & $-g^{\prime}(0)$ \\
\hline$[2 / 2]$ & 1.65812 & -0.712958 \\
{$[3 / 3]$} & 1.65812 & -0.700015 \\
{$[4 / 4]$} & 1.66033 & -0.700577 \\
{$[5 / 5]$} & 1.66026 & -0.700351 \\
{$[6 / 6]$} & 1.66079 & -0.700401 \\
{$[7 / 7]$} & 1.66075 & -0.700364 \\
{$[8 / 8]$} & 1.66090 & -0.700371 \\
{$[10 / 10]$} & 1.66093 & -0.700361 \\
{$[13 / 13]$} & 1.66095 & -0.700353 \\
{$[14 / 14]$} & 1.66095 & -0.700353 \\
{$[15 / 15]$} & 1.66095 & -0.700353 \\
\hline
\end{tabular}

Table 2. Values of skin friction coefficient $C_{f} R_{\boldsymbol{e}_{x}}^{\frac{1}{2}}$ for $K=0$, 1,2 and $R=0,1,2$, at $n=0$ and $n=0.5$ for different values of $\lambda$.

\begin{tabular}{ccccl}
\hline$\lambda$ & $K=0, R=0$ & $K=1, R=1$ & $K=2, R=2$ & $n$ \\
\hline 0.01 & 100.90 & 201.804 & 302.712 & 0 \\
0.02 & 50.901 & 101.808 & 152.721 & 0 \\
1.00 & 1.8601 & 3.8574 & 5.9401 & 0 \\
2.00 & 1.1523 & 2.8820 & 6.2011 & 0 \\
3.00 & 3.2552 & 24.224 & 82.713 & 0 \\
4.00 & 20.0981 & 176.23 & 559.46 & 0 \\
0.01 & 100.90 & 201.804 & 302.712 & 0.5 \\
0.02 & 50.901 & 101.808 & 152.721 & 0.5 \\
\hline
\end{tabular}

shrinking sheet [7]. The magnitude of microinertia $g$ for different values of $R$ and $K$ are plotted in Figures 6 and 7. It is observed that the magnitude of $g$ decreases with the increase in $R$ and increase with the increase in $K$, thus we also say that the behavior of $R$ and $K$ on $g$ is opposite but the boundary layer thickness reduces for both the cases. The velocity field $f$ for different values of $R$ and $K$ when $n=1 / 2$ is plotted in Figures 8 and 9. It is seen that the behavior of velocity 

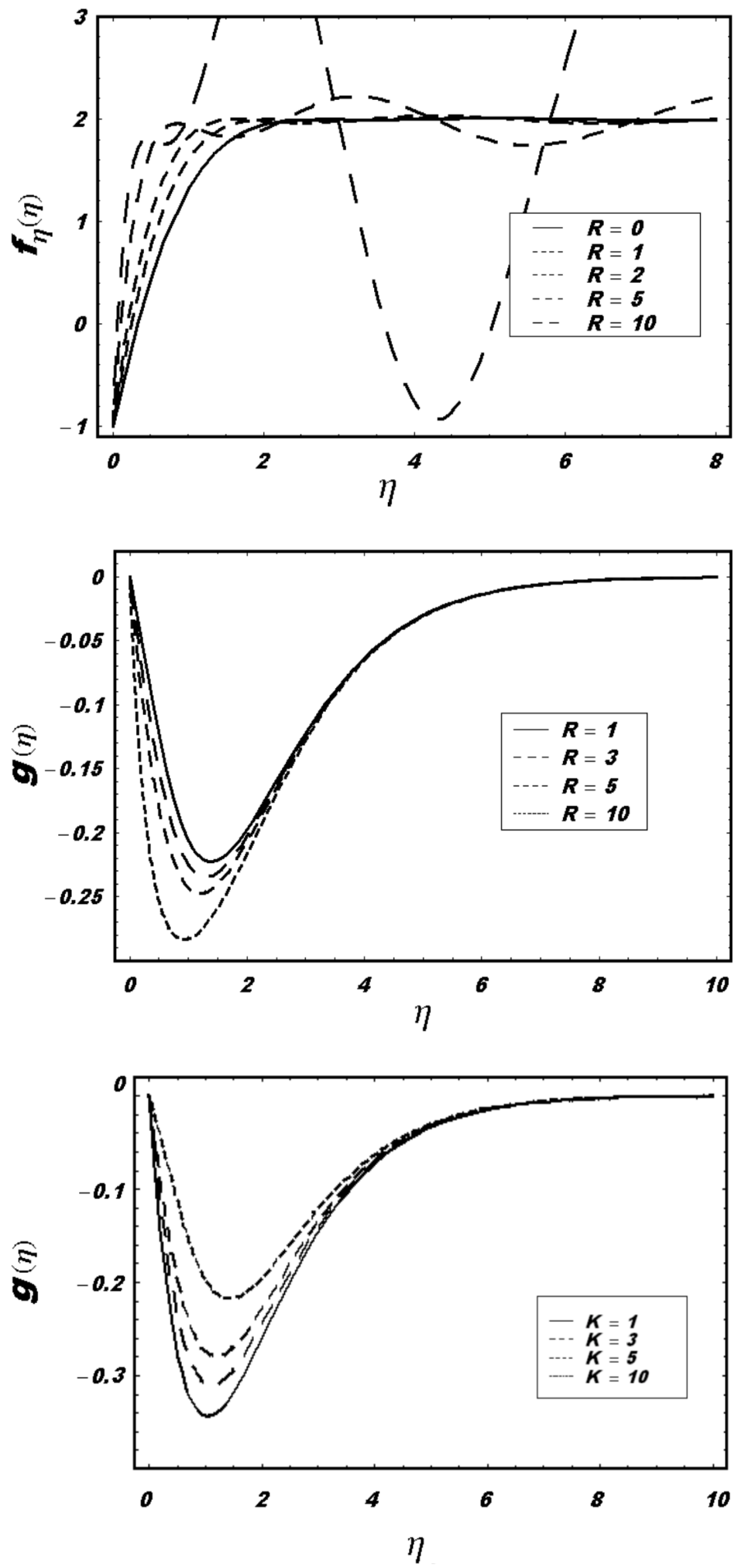

Fig. 5. Velocity field for different values of $R$ when $K=1, \lambda=2$, and $n=0$.

Fig. 6. Velocity field for different values of $R$ when $K=1$ and $n=0$.

Fig. 7. Velocity field for different values of $K$ when $R=1$ and $n=0$. 

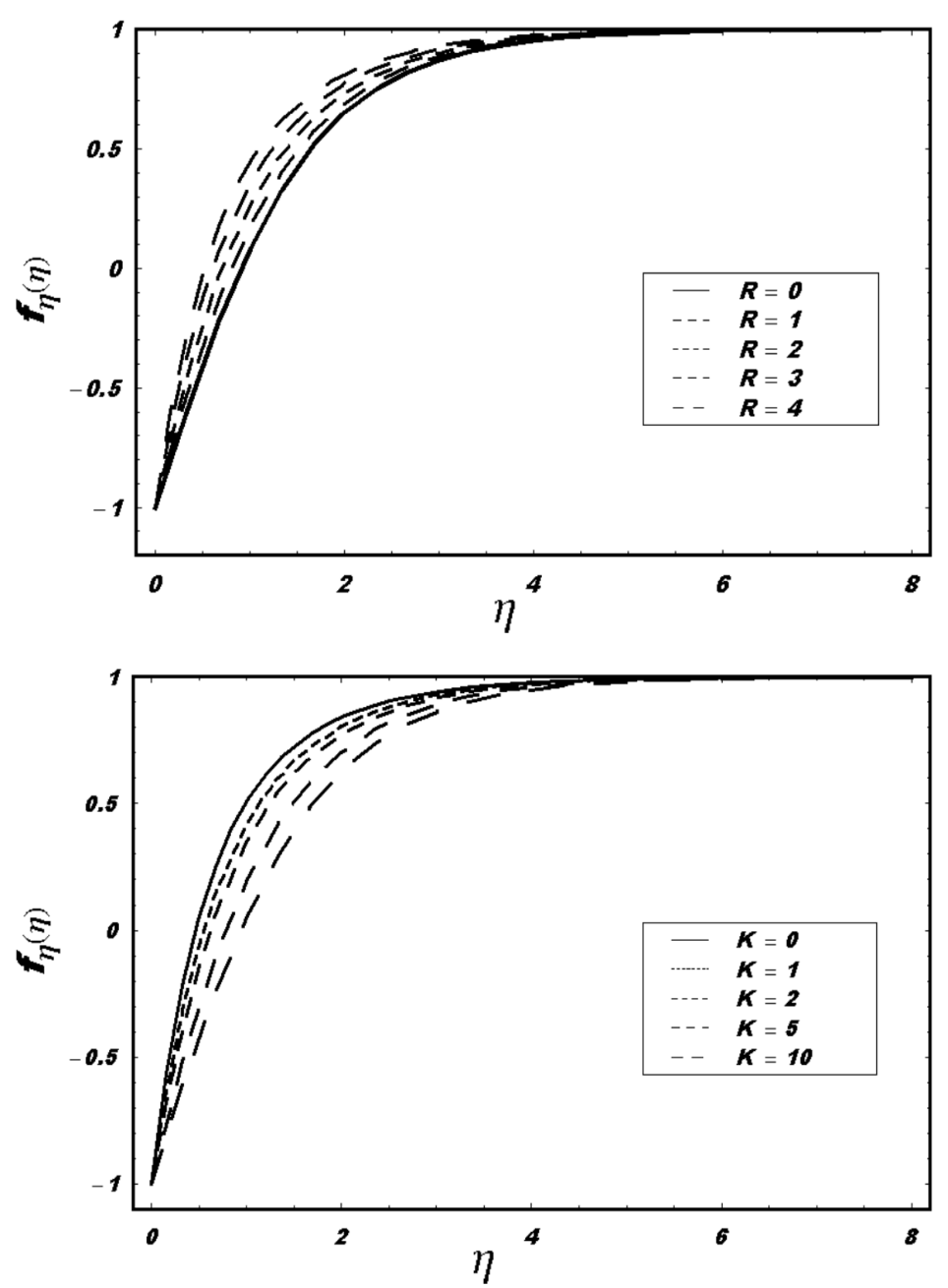

Fig. 8. Velocity field for different values of $R$ when $K=1$ and $n=1 / 2$.

Fig. 9. Velocity field for different values of $K$ when $R=1$ and $n=1 / 2$. for weak concentration is almost the same as discussed already for the strong concentration case.

[1] T. R. Mahapatra and A. S. Gupta, Acta Mechanica 152, 191(2001).

[2] R. Nazar, N. Amin, D. Filip, and I. Pop, Int. J. Engng. Sci. 42, 1241 (2004).

[3] Y. Y. Lok, N. Amin, and I. Pop, Int. J. Thermal Sci. 45, 1149 (2006).

[4] Y. Lok, N. Amin, and I. Pop, Int. J. Heat Mass Transf. 50, 4855 (2007).

[5] C. Y. Wang, Int. J. Engng. Sci. 46, 391 (2008).

[6] C. Y. Wang and M. Miklavic, Quarterly of Appl. Math., 64, 283 (2006).

\section{Acknowledgements}

The authors are thankful to the anonymous referees and Prof. Tasawar Hayat for useful discussion of the paper.

[7] C. Y. Wang, Int. J. Nonlin. Mech. 43, 377 (2008).

[8] T. Hayat, Z. Abbas, T. Javed, and M. Sajid, Chaos, Solutions, and Fractals 39, 1615 (2009).

[9] S. Nadeem and M. Awais, Phys. Lett. A 372, 4965 (2008).

[10] C. Y. Wang, European. J. Mech. B/Fluids 27, 678 (2008).

[11] A. C. Eringen, J. Math. Mech. 16, 1 (1966).

[12] S. Abbasbandy, M. Yurusoy, and M. Pakdemirli, Z. Naturforsch. 63a 564 (2008).

[13] S. Abbasbandy, Appl. Math. Model. 32, 2706 (2008). 
[14] S. J. Liao, Boca Raton: Chapman \& Hall/CRC Press (2003).

[15] H. Xu, S. J. Liao, and I. Pop, Acta Mechanica. 184, 87 (2006).

[16] T. Hayat and T. Javed, Phys. Lett. A 370, 243 (2007).

[17] T. Hayat, T. Javed, and Z. Abbas, Nonlinear Anal. Real World Applications 10, 678 (2008).

[18] A. C. Eringen, Microcontinuum Field Theories, II: Fluent Media, Springer, New York 2001.

[19] S. J. Liao, J. Fluid Mech. 488, 189 (2003).

[20] S. J. Liao and I. Pop, Int. J. Heat Mass Transf. 47, 75 (2004).

[21] T. C. Chiam, J. Appl. Math. Mech. (ZAMM) 62, 565 (1982).

[22] G. Lukaszewicz, Micropolar Fluids Theory and Application, Birkhauser, Basel 1998.

[23] J. Peddieson, Int. J. Engng. Sci. 10, 23 (1972).
[24] K. Vajravelu and J. R. Canon, Appl. Math. Comut. 181, 609 (2006).

[25] S. Abbasbandy, Phys. Lett. A 360, 109 (2006).

[26] Y. Tan and S. Abbasbandy, Com. Nonlinear Sci. Num. Sim. 13, 539 (2008).

[27] M. Sajid, T. Hayat, and S. Asghar, Nonlinear Dyn. 50, 27 (2007).

[28] R. S. R, Gorla, Int. J. Engng. Sci. 28, 145 (1990).

[29] A. H. Nayfeh, Perturbation methods, Wiley, New York 2000.

[30] S. J. Liao, Comput. Mech. 20, 530 (1997).

[31] S. J. Liao, Com. Nonlinear Sci. Num. Sim. 14, 983 (2009).

[32] S. J. Liao and Y. Tan, Stud. Appl. Math. 119, 297 (2007).

[33] D. A. S. Rees and I. Pop, IMA J. Appl. Math. 61, 179 (1998). 